\title{
Single-molecular fluorescence spectroscopy in protein folding: a theoretical modeling of multi-color experiments
}

\author{
V.A. Andryushchenko ${ }^{1,2}$, A.Yu. Palyanov ${ }^{1,3}$, S.F. Chekmarev ${ }^{1,2 *}$ \\ ${ }^{1}$ Novosibirsk State University, Novosibirsk, Russia \\ ${ }^{2}$ Institute of Thermophysics SB RAS, Novosibirsk, Russia \\ ${ }^{3}$ Institute of Informatics Systems SB RAS, Novosibirsk, Russia \\ *e-mail: chekmarev@itp.nsc.ru
}

Key words: protein folding, single-molecular fluorescence spectroscopy, molecular dynamics, collective variables, free energy surfaces

Motivation and Aim: The single-molecular fluorescence spectroscopy methods, such as the Förster Resonance Energy Transfer (FRET) and Photoinduced Electron Transfer (PET), have become a powerful tool to study protein folding. Currently, the donor and acceptor are typically positioned at the ends of the protein chain $[1,2]$. The results of the measurement are presented in the form of one-dimensional (1D) free energy profile along a reaction coordinate connecting the unfolded and native states of the protein, which allows one to see how the protein folds. However, since the protein usually follows a variety of essentially different folding pathways, in which case the folding kinetics are often very complex, such 1D profiles do not give a reasonably complete description of the folding process. At the same time, the donor and acceptor can be placed not only at the ends of the protein but also within the protein chain, so that a multi-color signal coming from different mutual positions of a set of donors and acceptors can be recorded [3]. In this case, two-dimensional (2D) free energy surfaces (FESs) can be constructed, which provide incomparably richer information about the folding process than the $1 \mathrm{D}$ profiles do. In the present work, using molecular dynamics simulations, we examine what information can be obtained if the fluorescence signal is monitored for two sets of donors and acceptors, and how the picture of folding thus obtained is complete in comparison to an "ideal" choice of collective variables to characterize the folding process. Methods and Algorithms: Since our goal was to understand the situation in general, we used a coarse-grained protein representation, i. e., each protein residue was represented by a bead placed at the position of the $\mathrm{C}_{\alpha}$-atom. The simulations were performed with molecular dynamics methods. Using the commonly employed collective variables, such as the radius of gyration and the RMSD from the native state, a "theoretical" FES was constructed for each protein, which was supposed to give a best representation of the folding process. To construct the corresponding "experimental" FES, two pairs of donors and acceptors were chosen, for which the characteristics relevant to the FRET and PET experiments were monitored. The donors and acceptors were represented by selected residues (beads).

Results: Folding of two proteins, BBL domain and GB1 protein, has been studied. The comparison of the "experimental" and "theoretical" FESs has shown that in contrast to the 2D surfaces (multi-color experiments), the 1D free energy profiles (single-color experiments) do not necessarily distinguish the essential protein states. The single-color experiments can, however, be successful at a suitable location of donor and acceptor, in particular, when they are located at the protein termini, as in the FRET-experiments on BBL folding [4].

Acknowledgement: This work was supported by Russian Foundation for Basic Research, grant No. 18-04-00013.

\section{References}

1. Neuweiler H., Johnson C.M., Fersht A.R. (2009) Proc. Natl. Acad. Sci. USA 106:18569.

2. Chung H.S., McHale K., Louis J.M., Eaton W.A. (2012) Science 335:981.

3. Lerner E., Cordes T., Ingargiola A., Alhadid Y., Chung S.Y., Michalet X., Weiss S. (2018) Science 359:288.

4. Liu J., Campos L.A., Cerminara M., Wang X., Ramanathan R., English D.S., Muñoz V. (2012) Proc. Natl. Acad. Sci. USA. 109:179. 